\title{
DER ZEITSCHRIFTTITEL.
}

Da mit dem Augenblicke, wo eine Namensänderung (ler Deutschen Elektrochemischen Gesellschaft oder selbst nur die geplante Erweiterung ihrer Ziele beschlossen wird, auch sofort eine Titeländerung unserer Zeitschrift notwendig wirl, so erscheint es angebracht, zugleich mit obigen Ausführungen von Geheimrat Ostwald die Diskussion hieriber anzuregen.

Für den etwaigen neuen Titel kommen meines Erachtens drei Gesichtspunkte in Betracht: I. Die Prägnanz, 2. die zutreffende Bezeichnung des Inhalts, 3. die Kontinuität mit der Vergangenheit, ausserdem noch einiges weniger Wichtige, was jeder von selbst im gegebenen Falle herausfühlen wird.

Der Titel "Berichte der deutschen BunsenGesellschaft", abkürzbar in "Bunsen-Ber.", würde für den Fall der Namensänderung dem Wunsch nach Präguanz entsprechen. Statt „Berichte" würde noch etwa an "Arbeiten", "Abhandlungen", "Annalen", "Verhandlungen" gedacht werden können, doch decken diese teils die Sache nicht vollkommen, teils klingen sie in Anbetracht des ephemeren Teils des Zeitschriftinhalts vielleicht zu anspruchsvoll.

Für eine dentliche Bezeichnung des Inhalts wïrde hiermit aber nicht genügend gesorgt sein; denn namentlich den Kreisen der Technik gegenüber, welche durch die Erweiterung des Programms für unsere Ziele interessiert werden sollen, ist eine Angabe des Arbeitsfeldes "angewandte physikalische Chemie, speziell Elektrochemie" oder, Elektrochemie und angetwandte physikalische Chemie" gewiss nützlich. Der Zusatz "Elektrochemie" erscheint namentlich im Interesse der Kontinuität erwünscht, und so würden sich alle drei Wünsche vielleicht durch folgende beiden Doppeltitel erfuillen lassen, die hiermit zur Diskussion gestellt seien:

\section{1. „Berichte der Deutschen Bunsen- Gesellschaft"}

mit dem Untertitel:

„Zeitschrift für Elektrochemle und angewandte resp. physikalische Chemie",

\section{"Zeitschrift für angewandte physikalische Chemie, Insbesondere Elektrochemie",}

oder:

2. "Zeitschrift für Elektrochemie

und angewandte physikalische Chemie". mit dem Untertitel:

\section{"Berichte der Deutschen Bunsen-Gesellschaft".}

In beiden Fällen wäre die wünschenswerte Möglichkeit vorhanden, wenu auf dem Titelblatt kein erheblicher typographischer Unterschied zwischen beiden Titeln gemacht wird, die Citatabkürzung ,Bunsen-Ber." $z u$ wählen, und dies ist sowohl wegen der Kürze wie wegen der Unterscheidung von der ,Elektrochemischen Zeitschrift" vorteilhaft.

Im Falle nur die Erweiterung des Arbeitsprogramms ohne Namensänderung beschlossen werden sollte, kätne yatürlich allein der Vorschlag 2. ohne den Untertitel in Betracht, und beim Citieren bliebe alles beim alten. Anderen Vorschlägen in dieser wichtigen Frage wird die Zeitschrift gern Raum geben und bittet um solche, sei es mit oder ohne Begründung der Formulierung.

R. A begg.

\section{VEREINSNACHRICHTEN.}

\section{Deutsche Elektrochemische Gesellschaft.} Hauptversammlung in Würzburg 1902.

Unter Hinweis anf die Mitteilungen in früheren Heften sind ergänzend und erweiternd folgende Vorträge angemeldet:

8. Hantzsch-Würzhurg: Ueber Isometie-Erscheinungen bei Salzen.

24. Billitzer-Göttingen: Elektrische Doppelschicht und absolutes Potential.

Anmeldungen zur Mitgliedschaft sind satzungsgemäss an den ersten Vorsitzenden, Ferrn Professor Dr. van't Hoff, Charlottenburg, Uhlandstr. 2, zu richten; die Anmeldungen müssen von einem Mit. glied der Gesellschaft befiurwortet sein.

Zahlungen werden ausschliesslich an den Schatzmeister, Herrn Dr. Marquart, BettethausenCassel, erbeten.

Alle anderen geschäftlichen Mitteilungen wolle man an die Geschäftsstelle der Deutschen Elektrochemischen Gesellschaft, Jeipzig, Mozartstr. 7 , richten.

Die Versendung der Vereinszeitschrift geschieht durch die Verlagsbuchhandlung unter deren Verantwortlichkeit, und zwar nach dem Auslande unter Kreuzband, nach dem Inlande ohne solches, wie bei
Tageszeitungen. Die Nachlieferung fehlender Nummern ist deshalb in Inlande möglichst bald bei der BestellPostanstalt nachzusuchen, ebenso ist diese - wie auch die Geschäftsstelle - von Adressenänderungen zu benachrichtigen. Etwaige weitere Mitteilungen über feblende Hefte, insbesondere vom Auslande, sind an die Geschäftsstelle zu richten.

An die neu eintretenden Herren Mitglieder wird die Vereinszeitschrift erst nach Zahlung des Mitgliedbeitrages geliefert.

\section{Anmeldungen für die Mitgliedschaft}

Gemäss \$ 3 der Satzungen werden hiermit die Namen der Herren, Firmen u.s. w., welche sich beim Vorstande für die Aufnabme gemeldet haben, veröffentlicht. Einspruch gegen die Aufnahme eines gemeldeten Mitgliedes ist innerhalb zweier Wochen (also bis zum 2o. März einschliesslich) $z u$ erheben.

Nr. 789. Reuter, Max, Dr, phil., Baden bei Wien, Franzenstrasse 17; durch Julius Friedrich Sacher.

\section{Adressenänderungen.}

Nr. 5ro. von Wirkner, Schalke in Westfalen, Kaiserstrasse 3 .

" 805. Zehrlaut, jetzt: Mainz, Kaiserstrasse 3 I. 